\title{
Giant multiple and bilateral presacral Tarlov cysts mimicking adnexal mass - imaging features
}

This article was published in the following Dove Press journal: International Medical Case Reports Journal

\author{
Monique Boukobza' \\ Alexandre Roussel' \\ Pedro \\ Fernandez-Rodriguez' \\ Jean-Pierrre Laissy ${ }^{1-3}$ \\ 'Department of Radiology, Assistance \\ Publique-Hôpitaux de Paris, Bichat \\ Hospital, Paris, France; '2INSERM \\ UI I 48, Paris, France; ${ }^{3}$ University Paris \\ 7, Bichat Hospital, Paris, France
}

\begin{abstract}
Perineural or Tarlov cysts (TCs) are nerve root cysts. They are usually incidental findings on MRI and are most frequently observed in the sacral spine. A 49-year-old woman presented with lower abdominal discomfort for several months. Physical examination demonstrated a cyst-like mass. An ultrasonographic examination revealed a cystic mass near the right ovary. MRI (3-Tesla unit) showed multiple, bilateral, and almost symmetric giant TCs with presacral space and endopelvic extension and minime scalloping. TCs originated from the right and left L5, S1, S2, and S3 nerve root sleeves entered the presacral space and extended into the pelvis. Keywords: presacral mass, perineural cyst, giant schwannoma, adnexal disease, giant cyst
\end{abstract}

\section{Introduction}

Tarlov cysts (TCs), also known as perineural cysts, typically develop at the junction of the dorsal ganglion and spinal posterior nerve root and have mainly a sacral location. They are a common incidental finding on lumbosacral MRI. In most cases, they are small and asymptomatic.

Huge presacral TCs are exceptionally rare. The uncommon endopelvic extension of a giant TC can lead to misdiagnosis and unnecessary laparotomy. We have reported a case of giant, multiple, and bilateral Tarlov cysts, with endopelvic extension, paucisymptomatic, mimicking an adnexal mass in a 49-year-old woman.

\section{Case}

A 49-year-old woman, without past medical history, presented with lower abdominal discomfort for several months. Physical examination demonstrated a floppy, cyst-like mass. Clinical examination was otherwise normal. An ultrasonographic examination revealed normal uterus and ovaries and also a cystic mass containing free echo fluid separated from the right ovary.

Sacral MRI (3-Tesla Signa ${ }^{\circledR}$ Hdxt system; GE Healthcare, Milwaukee, WI, USA) revealed several huge, bilateral, and almost symmetric cystic dilatations originating from the right and left L5, S1, S2, and S3 nerve root sleeves consistent with perineural cysts, ie, TCs. The cysts were filled with fluid, showed the same signal intensity as that of CSF on T1- and T2-weighted images, and were observed to be oval and lobulated. The cysts entered the presacral space through the right and left, enlarged L5-S1, S1S2, S2-S3, and S3-S4 foramens, extending anteriorly into the pelvis (Figure 1A-D). Bone erosion - "scalloping" - was confined to posterior left S2 body.
Correspondence: Monique Boukobza Department of Radiology, Assistance Publique-Hôpitaux de Paris, Bichat Hospital, 46 rue Henri Huchard, Paris 75018, France

Email m.boukobza@orange.fr 
The endopelvic segment of the cysts measured up to 48 $\mathrm{mm}$ in the greatest dimension (left [L] L5; right [R]; and left $\mathrm{S} 1)$. The measurements of the other cysts were as follows: L5 (L), $42 \mathrm{~mm}$; S2 (R and L), $20 \mathrm{~mm}$; and S3 (R and L), $34.2 \mathrm{~mm}$. A total of 8 huge cysts were found in this patient.

TC at left L5 was adjacent to the left ovary (Figure 1E) and the TC at S3 protruded along the right side of the rectum. TCs at $\mathrm{S} 1$ and $\mathrm{S} 2$ levels exerted extrinsic mass effect on the adjacent sigmoid loop (Figure 1E and F). All cysts had a complex pattern of being multiloculated with numerous internal septations. Three-dimensional (3D) acquisition, allowing multiplanar reformation, gave numerous information on the relationship of the cysts with the root fibers and surrounding structures. There was no TC enhancement after gadolinium chelate contrast administration (Figure 1G). The patient was managed conservatively.

\section{Consent}

Written informed consent was obtained from the patient for the publication of this case report.

\section{Discussion}

TCs are more common in females than in males with a 2:1 ratio $^{1,2}$ and are symptomatic in $\sim 1 \%$ of cases. They are usually incidentally discovered while performing CT or MRI for back pain, sciatica, or various other indications. The most commonly occurring symptoms are low back pain, pelvic, perineal, sacral or lumbar pain, or sciatica S1, S2 or both. In $10 \%$ of cases, bladder, bowel, or sexual dysfunctions are present. ${ }^{3}$ They are revealed at an average age of $40.4 \pm 14.3$ years. ${ }^{1,3}$ Very rarely, the sacral TCs are responsible of a cauda equina syndrome. ${ }^{4}$

The sacral TCs are often multiple, as in our case, and most commonly found at the $\mathrm{S} 2$ or $\mathrm{S} 3$ level. A presacral location is rare. An endopelvic extension is uncommon, which is present in 5\% of cases. ${ }^{2}$ Large TCs are usually defined as cysts with $\geq 1.5 \mathrm{~cm}$ diameter. The largest and the most symptomatic TCs occur in the sacral region. Large TCs are rare and show enlargement of neural foramina and bone erosion. Bone erosion is usually more marked in case of large or huge TCs. There is a negative correlation between the maximum diameter of the cysts and their numbers. ${ }^{1}$

A correlation between the size of the TC and marked radicular deficits was found by Elsawaf et al, ${ }^{5}$ even if not "statistically significant".

However, there may be a lack of correlation between the size of the cyst and symptomatology, as in the present case, ${ }^{6}$
A

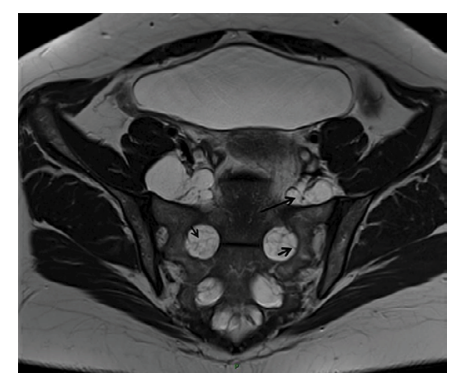

E

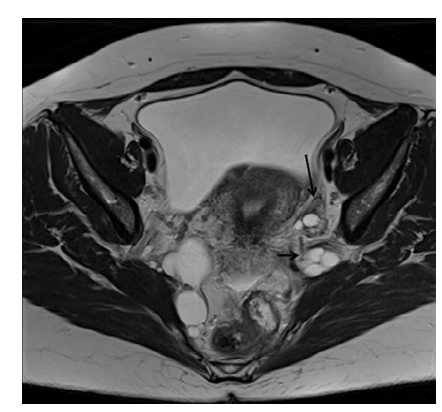

B

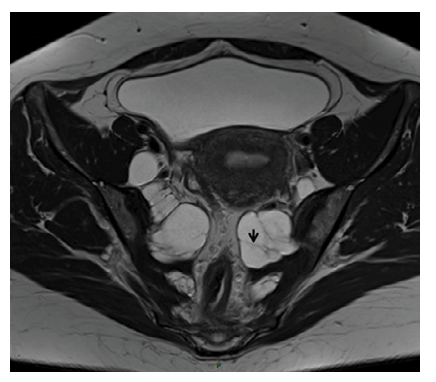

$\mathbf{F}$

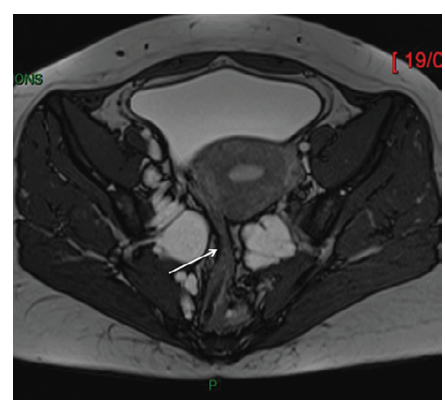

C

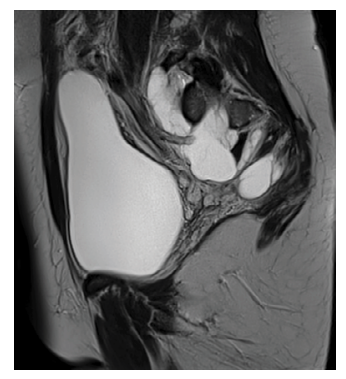

G

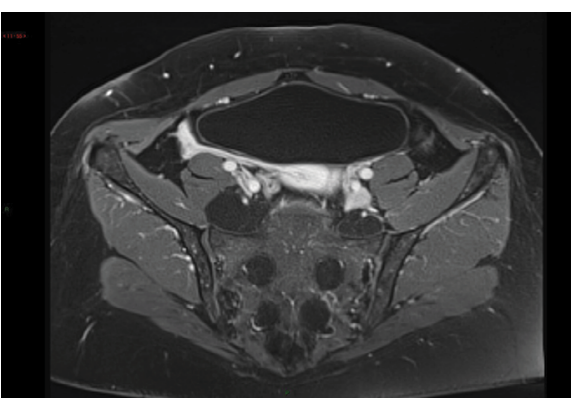

Figure I MRI of giant multiple Tarlov cysts. Axial (A and B), sagittal (C), and coronal (D) images: Multiformat Reformation (MPR) from 3 Dimensional TI MRI sequence. MRI revealed 8 Tarlov cysts (size: $30-63 \mathrm{~mm}$ ) multilocular with bone erosion of the left posterior $\mathrm{S} 2$ vertebral body. The development of the cysts is well visible on sagittal (C) and oblique (D) views. In (A) and (B), the cysts enter the presacral space through the enlarged right and left SI-S2, S2-S3, and S3-S4 foramina. The cysts are seen as homogenous hypointense signal masses on TI-weighted image and as hyperintense signal masses on T2-weighted image. All the cysts are multiseptated: the septa (A, B: short arrows) as the nerve roots (A, long arrow) coursing within are well identified on T2 images. A cyst (E, short arrow) is seen lateral to the left ovary (E, long arrow) and another one is responsible for a compression of the sigmoid (F, arrow). (G) The multiple cysts do not enhance after gadolinium chelate administration. The cysts enter the presacral space through the right and the left enlarged S1-S2, S2-S3, and S3-S4 foramina.

Abbreviations: MPR, multiplanar reformation; 3D, three-dimensional. 
but on the other hand, there are no differences of maximal diameter of the cysts between males and females.

Huge TCs, also called giant TCs as in the present case, are exceedingly rare and usually reported with this appellation when they have a maximum diameter of more than 3 $\mathrm{cm}$. Our review of the literature revealed 10 cases including the present case (Table 1). ${ }^{5,7-10}$ Out of 10 cases, 8 were female and the age of patients ranged from 7 to 67 years (mean: 34.7 years).

If excluding the case 2 of Elsawaf et $\mathrm{al}^{5}$ whose TC was diagnosed in a 7-year-old infant, the average age of adult patients was 37.7 years. In most reported cases, giant sacral TCs developed unilaterally (6 out of 10 cases) and were symptomatic ( 8 out of 10 cases) with pain being the most frequent symptom ( 6 out of 10 cases). ${ }^{7,10}$ Considering the number of affected levels in giant sacral TCs reported in the literature, we observed the following: 1 level: 5 patients; 2 levels: 1 patient; 3 levels: 3 patients; 4 levels: our case (Table 1). Finally 3 out of 10 cases of giant sacral TC mimicked adnexal masses (ie, 8, 9, and the present case).

In case of endopelvic extension, ${ }^{2}$ most cysts contain a homogeneous fluid collection that follows CSF signal on all MRI sequences, especially on T1- and T2-weighted images and a parietal course of the nerve roots is evidenced on MRI. Rarely, TCs present with a complex pattern of being multiloculated with numerous internal septations, which was demonstrated on T2-weighted images $\left(1.9 \%\right.$ for Kuhn et al $\left.{ }^{2}\right)$. It has to be emphasized that the dedicated sacral MRI gives better information than the standard lumbosacral MRI. ${ }^{3}$ Furthermore, 3D T2 acquisition allowing multiplanar reformation can give maximum information about the configuration of the cysts and their relationships. These information may be very useful if a surgical treatment is required. Finally, some authors consider that myelography can be useful to distinguish TCs from dural ectasias and arachnoid cysts. ${ }^{3}$

The TCs seen in our patient shared many uncommon features. The huge cysts were multiple, developed bilaterally (right and left from L5 to S3 roots) with almost equal size at each level and presented a complex pattern configuration. All the cysts were lobulated with numerous septa well visible on T2-weighted images and displayed endopelvic extension, despite our patient being paucisymptomatic.

Optimal treatment of symptomatic sacral TCs, if symptoms are consistent with cyst location, is still a subject of debate. Two types of treatments that are currently proposed are CT-guided percutaneous injection of fibrin sealant ${ }^{3}$ and microsurgical treatment. ${ }^{11,12}$

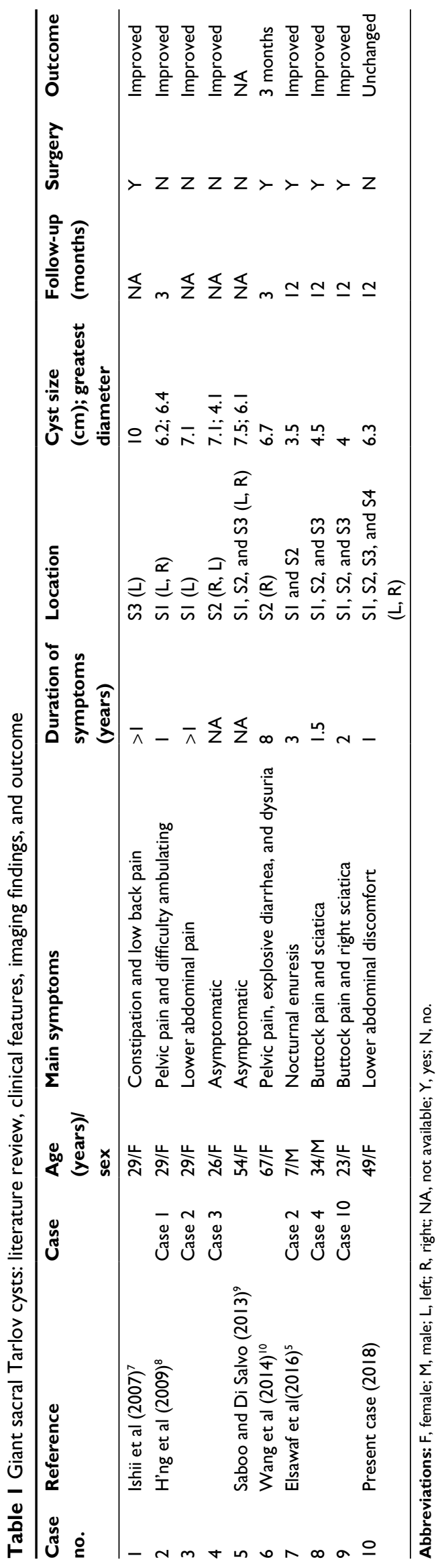




\section{Differential diagnosis}

When they are large, with presacral location and pelvic extension, TCs can mimic gynecological masses such as hydrosalpinx, ovaries cysts, and localized fluid due to adhesion on pelvic ultrasound. Our patient had no history of surgery or pelvic inflammatory disease; therefore, these two diagnoses were less considered.

The differential diagnosis is easy on pelvic ultrasound if the cystic lesion is multiseptated and separated from the ovaries, develops anteriorly to the sacrum, and is immobile on respiration. ${ }^{13}$

Only one case of giant presacral TC mimicking adnexal mass has been reported to date. ${ }^{7}$

MRI, giving good soft tissue resolution, multiplanar reconstruction, and absence of a bone interface, is the modality of choice. An enhancement of the cyst after contrast suggests a giant cystic sacral schwannoma or neurofibroma, a very rare entity that can mimic a TC. ${ }^{14}$ The association of a huge presacral meningocele with a TC has been reported in an isolated case. ${ }^{15} \mathrm{TCs}$ should be included in the differential diagnosis of presacral and endopelvic cystic masses. Dural ectasia that occurs with an increased incidence in Marfan syndrome may present as a huge presacral mass. But the radiological aspect is quite different as there is an expansion of the subdural space. However, a case of giant presacral TC occurring in a patient with Marfan syndrome has been reported. ${ }^{10}$

\section{Conclusion}

The reported case is a very uncommon presentation of TCs owing to 3 features: bilateral, almost symmetric huge TCs, and extending from L5 to S3 roots, with all these extending into the pelvis. Furthermore, very limited bone erosion was observed and few clinical symptoms were present.

This presentation mimicked adnexal mass on pelvic ultrasound examination. TCs must be considered in the differential diagnosis of such lesions, as well as sacral giant cystic schwannoma which must also be included in the differential diagnosis of giant presacral TC.

\section{Acknowledgment}

This case has not been presented at any conference and is not a part of a clinical trial.

\section{Disclosure}

The authors report no conflicts of interest in this work.

\section{References}

1. Sun JJ, Wang ZY, Teo M, et al. Comparative outcomes of the two types of sacral extradural spinal meningeal cysts using different operation methods: a prospective clinical study. PLoS One. 2013;8(12):e83964.

2. Kuhn FP, Hammoud S, Lefèvre-Colau MM, Poiraudeau S, Feydy A. Prevalence of simple and complex sacral perineural Tarlov cysts in a French cohort of adults and children. J Neuroradiol. 2017;44(1):38-43.

3. Murphy K, Oaklander AL, Elias G, Kathuria S, Long DM. Treatment of 213 patients with symptomatic Tarlov cysts by CT-guided percutaneous injection of fibrin sealant. AJNR Am J Neuroradiol. 2016;37(2):373-379.

4. Baker JF, Fitzgerald CW, O'Neill SC, McCormack D. Cauda equine syndrome secondary to bilateral sacral Tarlov cysts. Spine $J$. 2014;14(6):1065-1066.

5. Elsawaf A, Awad TE, Fesal SS. Surgical excision of symptomatic sacral perineural Tarlov cyst: case series and review of the literature. Eur Spine J. 2016;25(11):3385-3392.

6. Naderi S. Surgical approaches in symptomatic Tarlov cysts. World Neurosurg. 2016;86:20-21.

7. Ishii K, Yuzurihara M, Asamoto S, Doi H, Kubota M. A huge presacral Tarlov cyst. Case report. J Neurosurg Spine. 2007;7(2):259-263.

8. H'ng MW, Wanigasiri UI, Ong CL. Perineural (Tarlov) cysts mimicking adnexal masses: a report of three cases. Ultrasound Obstet Gynecol. 2009;34(2):230-233.

9. Saboo SS, Di Salvo D. Perineural cysts resembling complex cystic adnexal masses on transvaginal sonography. J Clin Ultrasound. 2013;41(1):55-58.

10. Wang B, Moon SJ, Olivero WC, Wang H. Pelvic pain from a giant presacral Tarlov cyst successfully obliterated using aneurysm clips in a patient with Marfan syndrome. J Neurosurg Spine. 2014;21(5):833-836.

11. Burke JF, ThawaniJP, Berger I, et al. Microsurgical treatment of sacral perineural (Tarlov) cysts: case series and review of the literature. J Neurosurg Spine. 2016;24(5):700-707.

12. Potts MB, McGrath MH, Chin CT, Garcia RM, Weinstein PR. Microsurgical fenestration and paraspinal muscle pedicle flaps for the treatment of symptomatic sacral Tarlov cysts. World Neurosurg. 2016;86(2):233-242.

13. Ghazizadeh S, Foss EW, Didier R, Fung A, Panicek DM, Coakley FV. Musculoskeletal pitfalls and pseudotumours in the pelvis: a pictorial review for body imagers. Br J Radiol. 2014;87(1042):20140243.

14. Attiah MA, Syre PP, Pierce J, Belyaeva E, Welch WC. Giant cystic sacral schwannoma mimicking Tarlov cyst: a case report. Eur Spine J. 2016;25(Suppl 1):84-88.

15. Hefti M, Landolt H. Presacral mass consisting of a meningocele and a Tarlov cyst: successful surgical treatment based on pathogenic hypothesis. Acta Neurochir (Wien). 2006;148(4):479-483.
International Medical Case Reports Journal

Publish your work in this journal

The International Medical Case Reports Journal is an international, peer-reviewed open-access journal publishing original case reports from all medical specialties. Previously unpublished medical posters are also accepted relating to any area of clinical or preclinical science. Submissions should not normally exceed 2,000 words or

\section{Dovepress}

4 published pages including figures, diagrams and references. The manuscript management system is completely online and includes a very quick and fair peer-review system, which is all easy to use. Visit $\mathrm{http}: / /$ www.dovepress.com/testimonials.php to read real quotes from published authors. 